\title{
Detection and localization of debonding damage in composite-masonry strengthening systems with the acoustic emission technique
}

\author{
E. Verstrynge \& K. Van Balen \\ KU Leuven, Department of Civil Engineering, Building Materials and Building Technology Division, \\ Heverlee, Belgium \\ M. Wevers \\ KU Leuven, Department of Metallurgy and Materials Engineering, Heverlee, Belgium \\ B. Ghiassi \& D.V. Oliveira \\ ISISE, University of Minho, Department of Civil Engineering, Guimarães, Portugal
}

\begin{abstract}
Different types of strengthening systems, based on fiber reinforced materials, are under investigation for external strengthening of historical masonry structures. A full characterization of the bond behavior and of the short- and long-term failure mechanisms is crucial to ensure effective design, compatibility and durability of the strengthening solution. In this paper, the effectiveness of the Acoustic Emission (AE) technique for debonding characterization and localization on Fiber Reinforced Polymer (FRP)- and Steel Reinforced Grout (SRG)-strengthened clay bricks is investigated. The AE technique proofs to be efficient for damage detection during accelerated ageing tests under thermal cycles and during experimental shear bond tests. AE data demonstrated the thermal incompatibility between brick and epoxy-bonded FRP composites during the accelerated ageing tests and debonding damage was successfully detected, characterized and located during the shear bond tests.
\end{abstract}

\section{INTRODUCTION}

Fiber reinforced materials are frequently used as externally bonded reinforcement for structural enhancement of concrete and masonry structures. They have well known advantages such as low weight to strength ratio and versatility in application. In recent years, composite materials such as fiber reinforced polymers (FRP) and steel reinforced grouts (SRG) have been under investigation for strengthening of (historical) masonry structures. Thereby, fully characterizing the bond behavior and failure mechanisms and studying the compatibility with the masonry substrate are crucial to ensure effective design and durability of the strengthening solution (Valluzzi et al., 2012). Aspects such as failure initiation, interfacial damage propagation, damage localization and long-term bond quality are still under investigation.

To ensure the development of efficient and durable FRP strengthening solutions, non-destructive techniques are essential for the following tasks:

- Characterization of the debonding mechanisms, to evaluate the efficiency of the applied strengthening technique and to support numerical modeling (parameter estimation and validation);

- Durability and compatibility assessment of the strengthening system (long-term behavior);

- Detection, localization and quantification of interfacial defects or progressive delamination for performance assessment, maintenance and earlywarning systems (on-site monitoring).
In this paper, the effectiveness of the acoustic emission (AE) technique for debonding characterization and localization on FRP- and SRGstrengthened clay bricks is investigated. The bond degradation will be analyzed with the $\mathrm{AE}$ technique during an accelerated ageing test under thermal cycles and during experimental shear bond tests. The different damage mechanisms that occur during a debonding process will be characterized and subsequent debonding areas will be located.

\section{DETECTION OF FRP DEBONDING}

\subsection{Characterization of debonding mechanisms}

Failure in FRP-strengthened masonry elements typically occurs due to FRP rupture or FRP debonding from the masonry substrate. Debonding in the masonry substrate, denoted as cohesive failure, occurs due to the lower mechanical properties of masonry compared to the repair material and the adhesive. Interfacial debonding, denoted as adhesive failure, normally occurs in case of poor surface preparation, e.g. when the surface is too smooth or wet upon application of the adhesive. It has been observed that environmental conditions, especially moist environments, can change the cohesive failure to adhesive failure. The tests described in this paper also indicated that specimens subjected to accelerated ageing 
tests are more likely to show adhesive failure. Also a combination of cohesive and adhesive failure surfaces, denoted mixed failure mode, can occur.

In case of strengthening with steel reinforced grout (SRG), in addition to masonry cohesive failure and adhesive debonding at the mortar-brick interface, debonding at the fiber-mortar interface can occur. The latter, being the most observed failure mode in the tests described in this paper, is followed by slipping of the fibers in the matrix.

\subsection{NDT for debonding detection}

Visual inspection and hammer tapping are the most widely used in-situ non-destructive testing methods for bond monitoring in FRP-strengthened elements, while several other methods are being applied such as digital image correlation (DIC), infrared (IR) thermography (Lai et al., 2012), ultrasonic testing (Mahmoud et al., 2010), shearography (Taillade et al., 2011) and acoustic emission (AE) testing.

DIC and IR thermography were applied during previous bond tests on similar specimens and setups. DIC has been used during shear bond tests on GFRP- and SRG-strengthened bricks to obtain the evolution of strains on the FRP surface (Ghiassi, Xavier, et al., 2013). The use of active IR thermography for detection of interfacial flaws and FRP delamination induced by environmental ageing, with specimens similar to the ones used in this study, is reported in (Ghiassi, Silva, et al., 2013).

\subsection{FRP debonding detection with $A E$}

For an introduction into the principles of $\mathrm{AE}$ testing in civil engineering (Grosse \& Ohtsu, 2008) and research on the application of this technique in masonry (De Santis \& Tomor, 2013; Verstrynge et al., 2009), the reader is referred to literature.

Limited results are reported in the literature regarding the analysis of debonding phenomena in externally strengthened masonry and concrete components by means of the acoustic emission technique. AE monitoring during FRP debonding from concrete beams and slabs was studied by (Carpinteri et al., 2007), who detected the propagation of flexural cracks in an FRP-strengthened beam, and by (Degala et al., 2009), who observed the progressive debonding of CFRP strips from concrete slabs and differentiated between CFRP debonding and concrete failure (flexural, compressive or shear failure) by looking at the relative intensity of the AE signals. Shear behavior of strengthened masonry walls was analyzed with the acoustic emission technique by (Masera et al., 2011) who observed decrease of the signal peak frequency upon failure of the masonry specimens. In the presented study, the debonding mechanism itself will be the object of investigation.

\section{EXPERIMENTAL PROGRAM}

The experimental study focuses on the detection of debonding with the $\mathrm{AE}$ technique during an accelerated ageing test under thermal fluctuations and during laboratory shear bond tests on two types of strengthening systems. Clay bricks were strengthened with Glass Fiber Reinforced Polymer (GFRP) and with Steel Reinforced Grout (SRG). Twelve single-lap shear bond tests were performed with AE detection, being three reference specimens and three aged specimens for each strengthening type.

\subsection{Materials and test specimens}

Test specimens consisted of single bricks strengthened with GFRP and SRG composites. Solid clay bricks with dimensions of 200x100x50 mm were used as substrate. The composite materials were cut in $50 \mathrm{~mm}$ width and applied to the bricks' surface along $150 \mathrm{~mm}$ length of the brick with a $40 \mathrm{~mm}$ unbonded part near the loaded end. GFRP strips were applied to the bricks' surfaces following the wet layup procedure. A two-part epoxy primer was applied for preparation of the substrate and a two-part epoxy resin was used as matrix for the GFRP.

For SRG-strengthened brick specimens, a 1directional medium density steel fiber net was used as reinforcement. The steel fibers were placed on a 3 mm thick layer of a lime-based mortar that was applied on the sand-blasted brick's surface. Then, another $3 \mathrm{~mm}$ mortar layer was applied to cover the steel fibers. Mechanical properties of the bricks and strengthening materials are presented in Table 1 as the mean value of five tests and the coefficients of variation $(\mathrm{CoV})$.

Table 1. Mechanical properties of strengthening material and bricks.

\begin{tabular}{lccc}
\hline Masonry brick & & Mean value & $\mathrm{CoV}(\%)$ \\
\hline Compressive strength & $f_{\mathrm{cb}}(\mathrm{MPa})$ & 14.2 & 15.7 \\
Flex. tensile strength & $f_{\mathrm{tb}}(\mathrm{MPa})$ & 1.6 & 24.6 \\
\hline GFRP strips & & & \\
\hline Tensile strength & $f_{\mathrm{tf}}(\mathrm{MPa})$ & 1250 & 15.0 \\
Elastic modulus & $E_{\mathrm{f}}(\mathrm{GPa})$ & 75.0 & 8.2 \\
Ultimate deformation & $\varepsilon(\%)$ & 3.0 & 20.2 \\
\hline Epoxy resin & & & \\
\hline Tensile strength & $f_{\mathrm{tm}}(\mathrm{MPa})$ & 53.8 & 9.7 \\
Elastic modulus & $E_{\mathrm{m}}(\mathrm{GPa})$ & 2.5 & 9.5 \\
\hline Primer & & & \\
\hline Tensile strength & $f_{\mathrm{tm}}(\mathrm{MPa})$ & 51.4 & 11.1 \\
Elastic modulus & $E_{\mathrm{m}}(\mathrm{GPa})$ & 2.4 & 6.1 \\
\hline Mortar & & & \\
\hline Compressive strength & $f_{\mathrm{cm}}(\mathrm{MPa})$ & 12.7 & 10.1 \\
\hline Steel fibers & & \\
\hline Tensile strength & $f_{\mathrm{ts}}(\mathrm{MPa})$ & 2980 & 2.9 \\
\hline
\end{tabular}




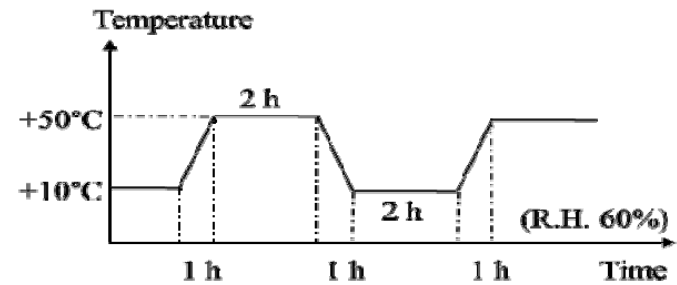

Figure 1. Hygrothermal exposure cycle

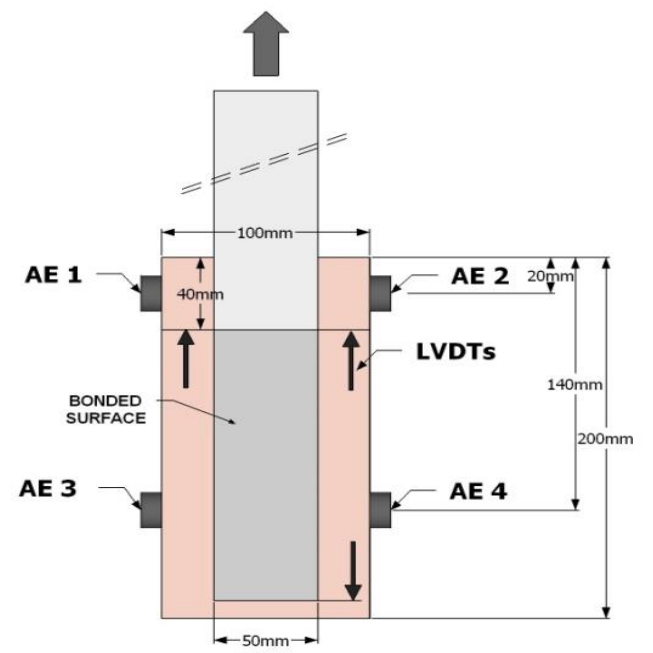

Figure 2. Single-lap shear bond test: test instrumentation and specimen dimensions.

\subsection{Experimental setup}

\subsubsection{Accelerated ageing tests}

To investigate the effect of environmental exposure, the specimens were exposed to 180 temperature cycles in a climatic chamber. In each cycle, the temperature was kept constant at $+10^{\circ} \mathrm{C}$ for $2 \mathrm{~h}$. It was then increased to $+50^{\circ} \mathrm{C}$ in $1 \mathrm{~h}$, followed by $2 \mathrm{~h}$ constant temperature at $+50^{\circ} \mathrm{C}$. Then, the temperature was decreased again to $+10^{\circ} \mathrm{C}$ in $1 \mathrm{~h}$, resulting in $6 \mathrm{~h}$ cycles of exposure, see Figure 1.

During the accelerated ageing process, AE hits were monitored on four specimens using a 4-channel Vallen AMSY-5 system with $150-500 \mathrm{kHz}$ operation frequency and $5 \mathrm{MHz}$ sampling rate. Four $150 \mathrm{kHz}$ resonance sensors were attached to the middle of the side of a brick by means of hot melt glue, which was chosen to resist temperatures of $50^{\circ} \mathrm{C}$ without softening. The preamplifier gain was set to $34 \mathrm{~dB}$ with a fixed threshold level of $50 \mathrm{~dB}$. To calculate the $\mathrm{AE}$ energy, the AE signal is squared and integrated and the energy unit (eu) is given by $1 \mathrm{eu}=10^{-14} \mathrm{~V}^{2} \mathrm{~s}$.

\subsubsection{Shear bond tests}

Single-lap shear bond tests were performed using a closed-loop servo-controlled testing machine with maximum load capacity of $50 \mathrm{kN}$. A rigid supporting steel frame was used to support the specimens and avoid misalignments in the load application. The specimens were pulled monotonically with a speed rate of $5 \mu \mathrm{m} / \mathrm{sec}$ under displacement control and the resulting load was measured by means of a load cell. The relative slip between the composite material and the brick was measured with two LVDTs glued at the loaded end and one LVDT glued at the free end, (Figure 2). Four $150 \mathrm{kHz}$ resonance AE sensors were attached two by two on opposite sides of the bricks.

To locate the AE sources in real time, a standard planar location algorithm is applied (Vallen Systeme $\mathrm{GmbH}, 2004)$, which assumes isotropic and homogeneous velocity of wave propagation and iterates until a minimum location error is obtained, based on the wave velocity, the sensor locations and the arrival time difference of the $\mathrm{AE}$ event at the different sensors. Setting the correct wave velocity is particularly difficult for the setup at hand, since the limited size of the specimens causes reflections and boundary effects and the mechanical properties of the involved materials, and thus the wave velocities, are not fully isotropic. In addition, the setup is in fact 3D (AE sensors are placed on the side of the specimen while cracks occur towards the front surface), while a planar sensor setup and location algorithm are applied; the wavelength is equal to the velocity/frequency ratio (approximately $1000 \mathrm{~m} / \mathrm{s} / 150$ $500 \mathrm{kHz}=2-7 \mathrm{~mm}$ ) and poses a lower limit for the location accuracy; Crack formation during the test will increase the heterogeneity of the specimen and hinder source location towards the end of the test.

Some of these issues can be solved by applying more advanced location algorithms and more accurate arrival time determination. Since the location accuracy is not the main focus of the present research, a pragmatic approach was followed for the calibration. A grid $(20 \times 30 \mathrm{~mm})$ is drawn on the back of the bricks and the wave velocity in each specimen is determined by searching for the minimum average source location error, by means of pencil lead breaks before the test. This resulted in an average location error limited to $12 \mathrm{~mm}$ in the middle area of the bricks and a location error between 5-17 $\mathrm{mm}$ for the middle point of the grid. This latter point has equal distance to all sensors, a large error at this location thus indicates a non-homogeneous specimen or a non-exact positioning of the AE sensors.

\section{RESULTS AND DISCUSSION}

\subsection{Damage detection during environmental ageing}

Damage progress was monitored by means of acoustic emission detection on two SRG- and two GFRPstrengthened bricks. The average number of AE hits per day recorded for each specimen within a period of 45 days is presented in Figure 3. Limited AE activity is recorded for each type of specimen and, as a first observation, it can be mentioned that not much difference is observed between the SRG- and GFRPstrengthened specimens. 


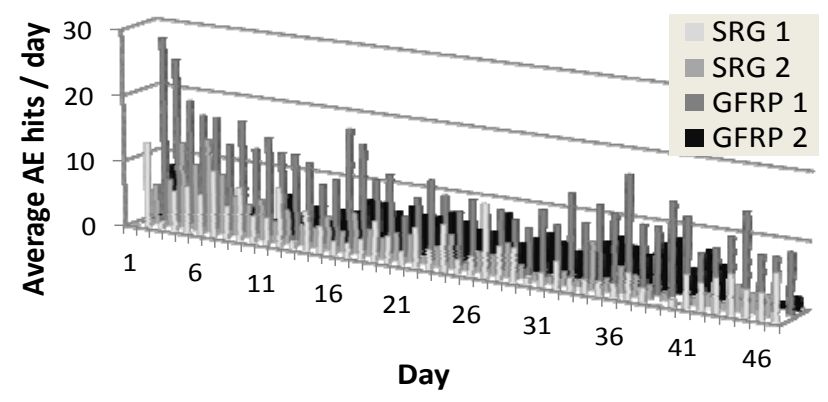

Figure 3. Average number of AE hits per day, recorded on 2 SRG-strengthened and 2 GFRP-strengthened specimens during environmental ageing test.
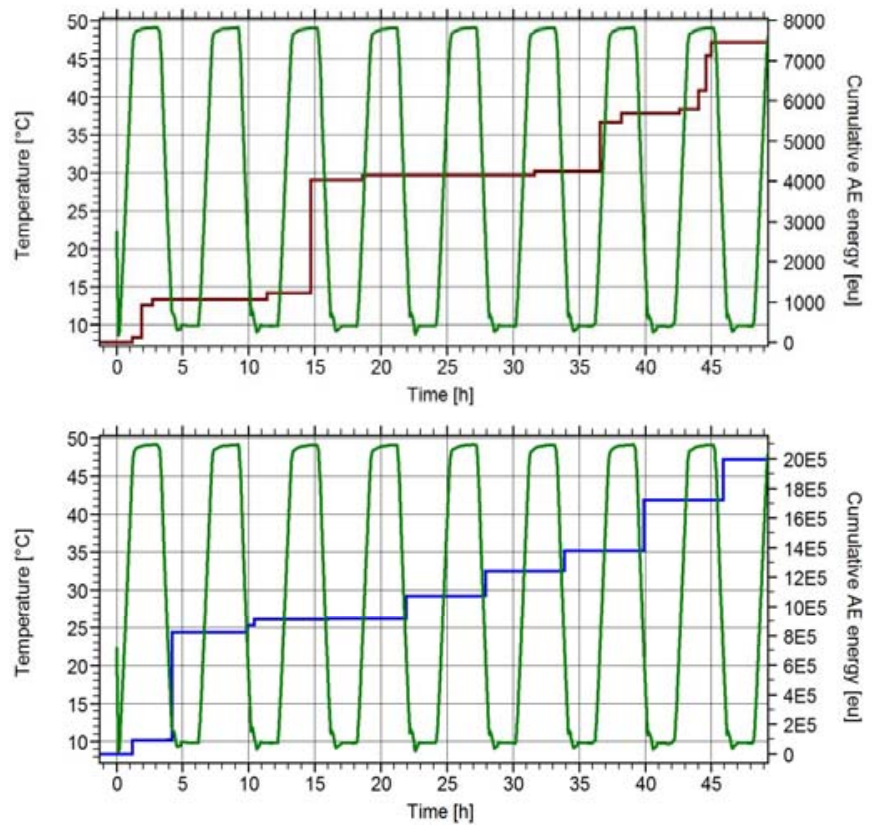

Figure 4. SRG-strengthened specimen: typical temperature fluctuation and random emission of AE energy (above); GFRPstrengthened specimen: typical temperature fluctuation and periodically emitted AE energy (below).

When the moments of AE energy emission are compared for both types of specimen, an important difference is observed. AE emissions in the SRGstrengthened specimens occur randomly, while for the GFRP-strengthened bricks, the majority of AE energy is emitted during temperature decrease (Figure 4). This is an indication of the different damage sources. The AE hits which are detected from SRGstrengthened specimens probably originate from the further hardening, shrinking or cracking of mortar. In the GFRP-strengthened bricks, the AE output during temperature decrease is a manifestation of the thermal incompatibility between the epoxy glue and the brick. Since the thermal expansion coefficient of epoxy can be up to 10 times larger compared to brick, the temperature cycling causes stress concentrations which might lead to damage propagation at the brick-GFRP interface.

\subsection{Damage detection during shear bond tests}

Typical AE results obtained from the debonding tests on GFRP-strengthened brick specimens are presented in Figures 5-6. The results in Figure 5 are presented for a specimen with mixed cohesive/adhesive failure mode, in terms of cumulative AE energy and slip development during the test. Generally, the debonding phenomenon can be divided into three main regions: elastic range, microcracking range, macro-cracking and progressive fracture. In the elastic range, the system deforms without any crack generation or AE activity. The small displacement measured at this stage is due to the elastic deformation of the FRP composite. As the applied force increases, micro-cracks appear in the interfacial region and they can be distinguished by initiation of AE activity with low emitted energies. As the debonding progresses, macro-cracks are formed and propagate along the interface with higher fracture energy being released. The cumulative AE energy increases with a stepwise pattern in which each sudden jump can be attributed to macrofracture events. A sudden release of AE energy is also observed at the moment of full debonding at the end of the test. The cumulative AE energy could thus be applied to define the subsequent regions of fracture progress.

The effect of failure mode on the AE outputs is investigated in Figure 6. A clear distinction is found between $\mathrm{AE}$ outputs of specimens with different failure modes. In the specimen with cohesive debonding, the AE energy release remains relatively low throughout the test, accompanied by a sudden and large amount of AE energy release when debonding occurs at the end of the test. The observed behavior confirms the brittle and sudden nature of the cohesive debonding. In the specimen with cohesive/adhesive failure, a progressive release of energy is observed during the test. In the specimen with adhesive debonding mode, progressive detection of $\mathrm{AE}$ energies is observed until complete debonding. However, the magnitude of the detected energies is much lower than the ones detected in the specimens with cohesive failure mode, due to the different nature and fracture properties of brick and FRP/brick interface.

Figure 7 presents a typical result obtained from an SRG-strengthened brick specimen, which failed with slipping of the steel fibers and mortar cover separation. Mainly, three regions representing different mechanisms can be observed during the debonding process. AE activities before the first cracking of the mortar are negligible. During the mortar cracking, the rate of $\mathrm{AE}$ activities increases and high AE energy is detected as the force increases. The resisting mechanisms in this region are adhesive bond and friction between the steel fiber and mortar. Detachment of the bond is accompanied by 
releasing relatively high fracture energies, observed as sudden jumps in the AE cumulative energy curve. As the debonding progresses, the bond diminishes and friction governs the failure mechanism resulting in a reduction of the detected $\mathrm{AE}$ energy rate. The debonding occurs with a sudden force reduction and slip increase. In contrary to the GFRP-strengthened specimens, no direct relation can be observed between the measured slip and AE cumulative energy.

In Figure 8, a comparison is made between two SRG-strengthened specimens with different failure modes, namely brick/mortar detachment and fibers slipping. A brittle behavior is observed in the specimen with brick/mortar detachment failure. The detected AE energy level in this specimen is very low during the test followed by a sudden release of energy at the moment of debonding. On the other hand, the fibers slipping failure mode produces a progressive release of energy during the test while the adhesive bond diminishes, followed by a reduction of the $\mathrm{AE}$ energy rate in the stage governed by frictional resistance. A more detailed analysis and characterization of debonding mechanisms based on AE results was presented in (Ghiassi et al., 2014).

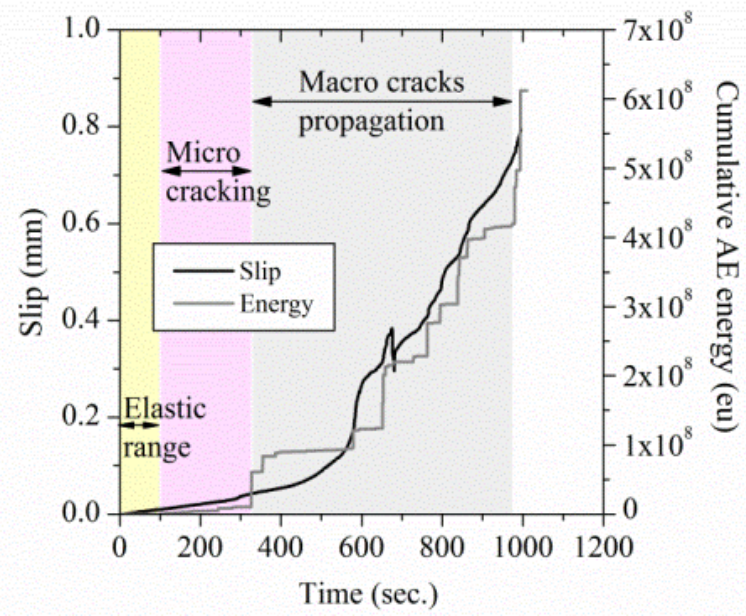

Figure 5. Typical AE results in a GFRP-strengthened brick specimen with cohesive/ adhesive failure mode: evolution of slip and cumulative AE energy

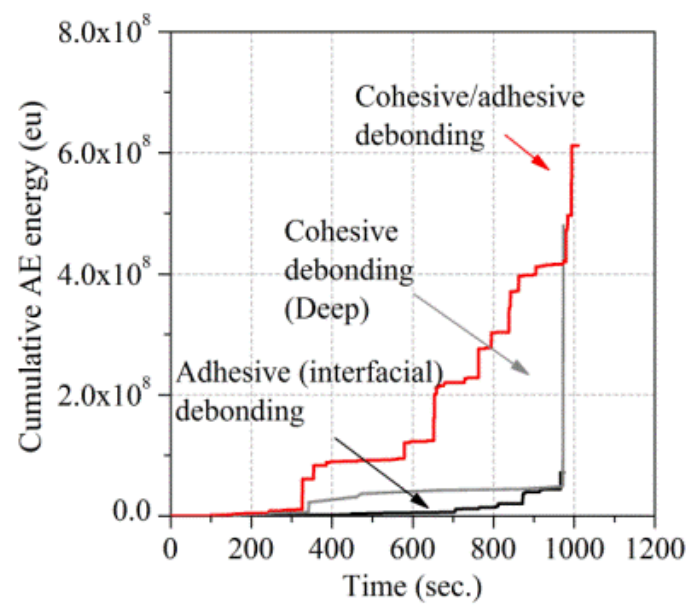

Figure 6. Comparison of AE output for different failure modes in GFRP-strengthened bricks

\subsection{Location of debonding}

During the single-lap shear bond tests, AE sources were located in real time. For the SRG-strengthened specimens, very few $\mathrm{AE}$ sources are located due to heterogeneity of the propagation path. For the GFRP-strengthened specimens, AE source location starts with the onset of the macro-fracture range at the loaded end of the laminate and progressively moves down during the test. This is in accordance with the expected downward movement of the debonded area.

This downshift of located AE events as a function of time is illustrated in Figure 9, which presents the total energy of all AE events located in zones of 1 $\mathrm{cm}$ perpendicular to the loading direction. The energy plots are made at specific time intervals, as indicated on the force-slip curves. Progressive debonding can more distinctively be observed for the specimen with predominantly cohesive debonding (=progressive cohesive/adhesive debonding) and the cohesive debonding phenomenon typically produces more and higher-energy AE sources.

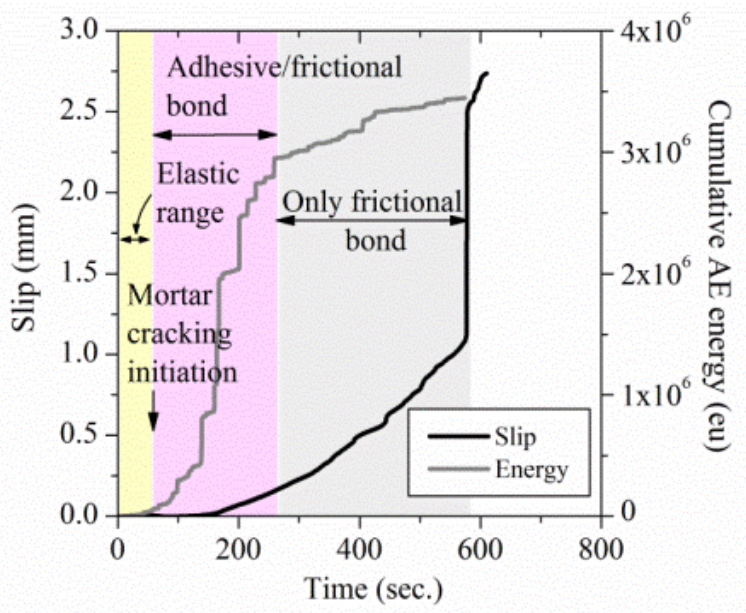

Figure 7. Typical AE results in an SRG-strengthened brick specimens: evolution of slip and cumulative AE energy

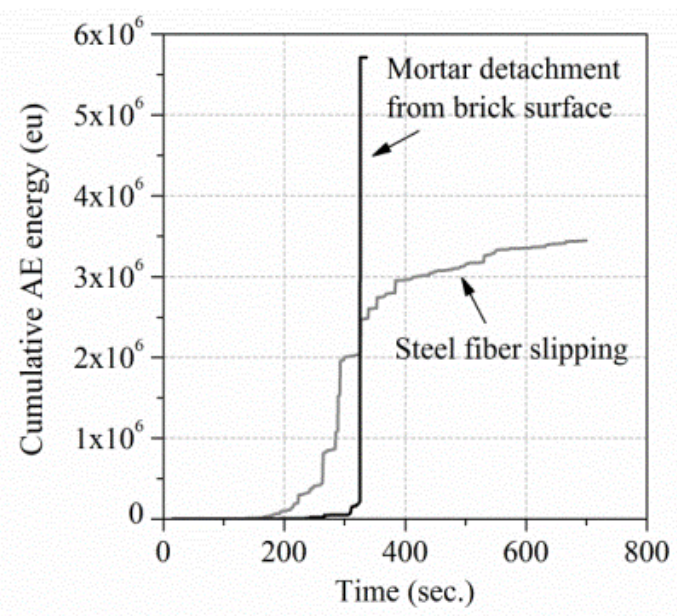

Figure 8. Comparison of AE output for different failure modes in SRG-strengthened bricks 

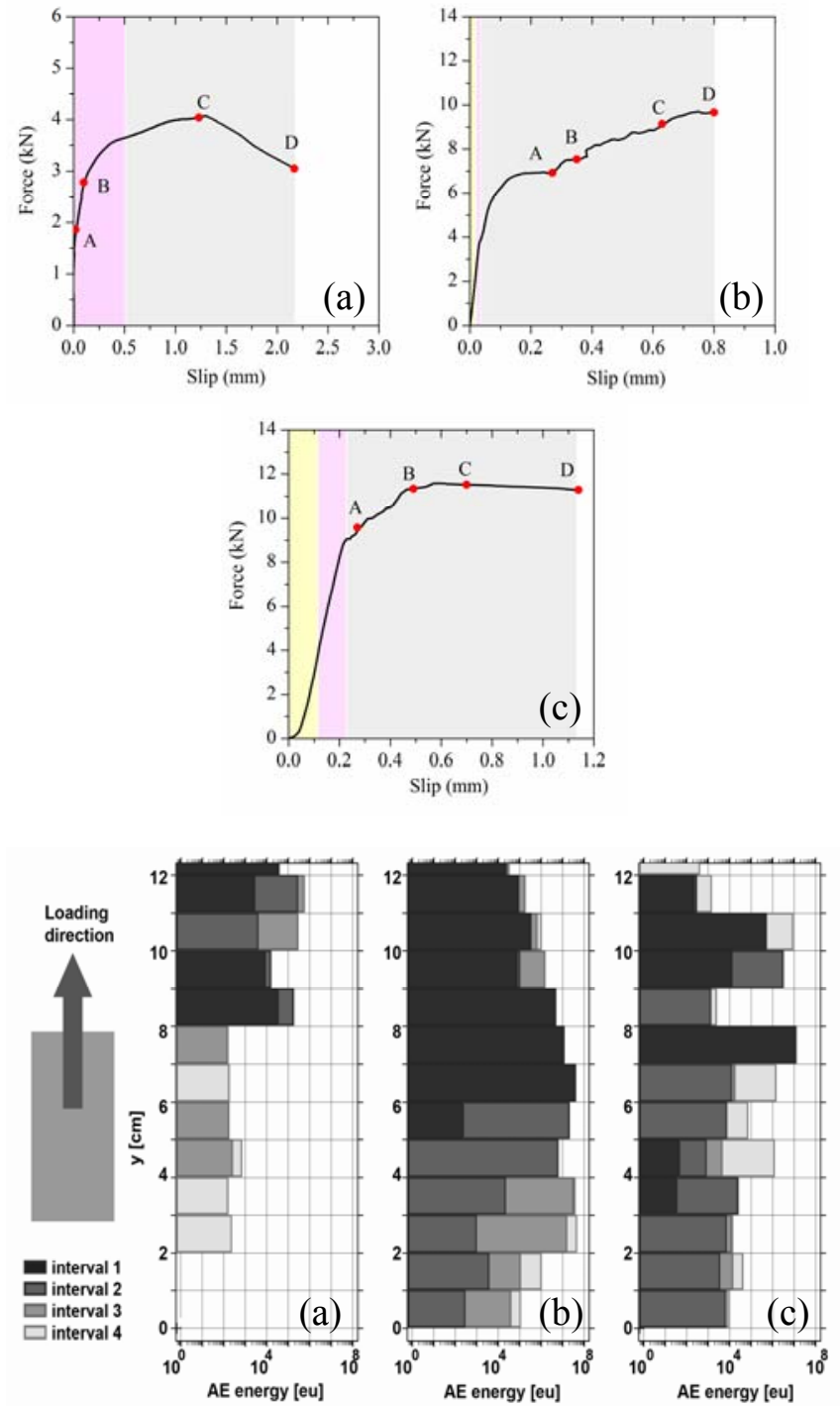

Figure 9. Cumulated energy of located AE events on SRGstrengthened specimen (a), GFRP-strengthened specimen with progressive cohesive/adhesive debonding (b) and with predominantly adhesive debonding (c). Time intervals 1 to 4 refer to the moments indicated on the force-slip curves (e.g. from the start to $\mathrm{A}$ is interval 1 , from $\mathrm{A}$ to $\mathrm{B}$ is interval 2).

\section{CONCLUSIONS}

Acoustic emission data obtained during the accelerated ageing test demonstrated the thermal incompatibility between the clay bricks and epoxy-bonded FRP composite, since AE hits were predominantly detected during temperature decrease for the GFRPstrengthened brick specimens. This conclusion strengthens the general consensus that besides epoxy-based systems, new strengthening techniques should be developed with better mechanical and thermal compatibility with the masonry substrate.

During the experimental shear bond tests, debonding damage was successfully detected, characterized and located, although location accuracy is limited due to the relative dimensions of the applied setup.

\section{ACKNOWLEDGEMENTS}

The authors acknowledge the financial support of the Research Foundation - Flanders (FWO) for the mobility grant offered to Els Verstrynge.

\section{REFERENCES}

Carpinteri, A., Lacidogna, G., \& Paggi, M. (2007). Acoustic emission monitoring and numerical modeling of FRP delamination in RC beams with non-rectangular crosssection. Materials and Structures, 40(6), 553-566.

De Santis, S., \& Tomor, A. K. (2013). Laboratory and field studies on the use of acoustic emission for masonry bridges. Ndt \& E International, 55, 64-74.

Degala, S., Rizzo, P., Ramanathan, K., \& Harries, K. A. (2009). Acoustic emission monitoring of CFRP reinforced concrete slabs. Construction and Building Materials, 23(5), 2016-2026.

Ghiassi, B., Silva, S.M., Oliveira, D., Lourenço , P.B., \& Bragança, L. (2013). Assessment of the bond quality degradation in FRP-strengthened masonry using IR thermography technique Paper presented at the FRPRCS11, Guimaraes, Portugal.

Ghiassi, B., Verstrynge, E., Lourenco, P. B., \& Oliveira, D. V. (2014). Characterization of debonding in FRPstrengthened masonry using the acoustic emission technique. Engineering Structures, 66, 24-34.

Ghiassi, B., Xavier, J., Oliveira, D.V., \& Lourenço, P.B. (2013). Application of digital image correlation in investigating the bond between FRP and masonry. Compos struct, 106, 340-349.

Grosse, C.U., \& Ohtsu, M. (Eds.). (2008). Acoustic emission testing - basics for research - applications in civil engineering: Springer.

Lai, W. L., Lee, K. K., Kou, S. C., Poon, C. S., \& Tsang, W. F. (2012). A study of full-field debond behaviour and durability of CFRP-concrete composite beams by pulsed infrared thermography (IRT). NDT \& E International, 52(0), 112-121.

Mahmoud, A.M., Ammar, H.H., Mukdadi, O.M., Ray, I., Imani, F.S., Chen, A., \& Davalos, J.F. (2010). Nondestructive ultrasonic evaluation of CFRP-concrete specimens subjected to accelerated aging conditions. NDT \& E International, 43(7), 635-641.

Masera, D., Bocca, P., \& Grazzini, A. (2011). Frequency Analysis of Acoustic Emission Signal to Monitor Damage Evolution in Masonry Structures. 9th International Conference on Damage Assessment of Structures (Damas 2011), 305.

Taillade, F., Quiertant, M., Benzarti, K., \& Aubagnac, C. (2011). Shearography and pulsed stimulated infrared thermography applied to a nondestructive evaluation of FRP strengthening systems bonded on concrete structures. Constr Build Mater, 25(2), 568-574.

Valluzzi, M.R., Oliveira, D.V., Caratelli, A., \& al., et. (2012). Round robin test for composite-to-brick shear bond characterization. J Mater Struct, 45, 1761-1791.

Verstrynge, E., Schueremans, L., Van Gemert, D., \& Wevers, M. (2009). Monitoring and predicting masonry's creep failure with the acoustic emission technique. NDT \& $E$ International, 42(6), 518-523. 